\title{
Enriched finite elements for branching cracks in deformable porous media
}

\author{
Mao Sheng ${ }^{\mathrm{a}}$, Gensheng $\mathrm{Li}^{\mathrm{a}, *}$, Subhash Shah ${ }^{\mathrm{b}}$, Anthony R. Lamb ${ }^{\mathrm{c}}$, \\ Stéphane P.A. Bordas ${ }^{\mathrm{d}, e, * *, 1}$ \\ a State Key Laboratory of Petroleum Resources and Prospecting, China University of Petroleum, Beijing 102249, China \\ ${ }^{\mathrm{b}}$ Well Construction Technology Center, University of Oklahoma, Norman, OK 73069, United States \\ ${ }^{\mathrm{C}}$ Intelligent Structural Integrity Management (IntelliSIMS), Houston, TX 77024, United States \\ ${ }^{\mathrm{d}}$ Research Unit in Engineering Science, Computational Mechanics, University of Luxembourg, Luxembourg City, Luxembourg \\ e Institute of Mechanics and Advanced Materials, Theoretical and Applied Mechanics, Cardiff University, Wales, United Kingdom
}

\section{A R T I C L E I N F O}

\section{Article history:}

Received 11 February 2014

Received in revised form

30 June 2014

Accepted 17 September 2014

\section{Keywords:}

Crack

Porous media

Finite element method

\begin{abstract}
A B S T R A C T
In this paper, we propose and verify a numerical approach to simulate fluid flow in deformable porous media without requiring the discretization to conform to the geometry of the sealed fractures (possibly intersecting). This approach is based on a fully coupled hydro-mechanical analysis and an extended finite element method (XFEM) to represent discrete fractures. Convergence tests indicate that the proposed scheme is both consistent and stable. The contributions of this paper include: (1) a new junction enrichment to describe intersecting fractures in deformable porous media; (2) the treatment of sealed fractures. We employ the resulting discretization scheme to perform numerical experiments, to illustrate that the inclination angles of the fractures and the penetration ratio of the sealed fractures are two key parameters governing the flow within the fractured porous medium.
\end{abstract}

(c) 2014 Elsevier Ltd. All rights reserved.

\section{Introduction}

Natural fractures are common in sedimentary rocks and can be either open or cemented. In fractured oil/gas reservoirs, connected and open fractures are the dominant cause for fluid transport. Neuman [1] and Berkowitz [2] offer a comprehensive review of experimental, modeling and numerical methods for fluid transport in geological media with opened fractures. However, previous work rarely tackled the impact of sealed fractures, which is an important part of the porous matrix in most rocks [3]. Mineral deposits are believed to be the main cause of sealing (see [4] for a geological view on this phenomenon). With the further development of shale gas exploration, which has been taking place over several decades already, sealed fractures will most likely attract a recrudescent interest because they can be potentially reopened to become active flow paths after hydraulic fracturing [5]. In shale

\footnotetext{
** Correspondence to: College of Petroleum Engineering, China University of Petroleum, 102249 Beijing, China.

** Corresponding author at: Research Unit in Engineering Science, Computational Mechanics, University of Luxembourg, Luxembourg City, Luxembourg.

E-mail addresses: ligs@cup.edu.cn (G. Li), bordasS@cardiff.ac.uk, stephane.bordas@alum.northwestern.edu (S.P.A. Bordas).

${ }^{1}$ ORCID ID 0000-0001-7622-2193.
}

deposits, sealed fractures usually have high length/width aspect ratios and tend to be cemented by silicate and calcite $[3,6]$. Since the minerals filling the fractures have a much lower permeability than the surrounding rock, these fractures can be considered as impermeable walls, through which no fluid exchange is possible. This assumption is supported by experimental data, such as magnetic resonance imaging [7]. Concisely, the flow disturbances engendered by sealed fractures strongly contribute to the heterogeneity of porous media, and quantifying their effect on the flow is of considerable geological relevance and can provide valuable information to engineers when predicting flow rates.

A discrete fracture, or crack, is often modeled as a mechanical discontinuity, across which the displacements and pore pressure are discontinuous. When these cracks propagate, these discontinuities evolve within the domain. Several classes of methods are available to discretize mechanics problems involving such evolving discontinuities. The most traditional is the finite element method (FEM), in which elements and nodes have to be placed along the crack surface and "special" elements are used to represent the crack tip singularity when the latter arises, e.g. when the bulk material is assumed linear elastic [8]. Other methods have emerged, based on the partition of unity method $[9,10]$, namely, the enriched/extended finite element methods (XFEM) [11,12], generalized finite element methods [13], the smoothed extended finite element method [14-17], the intrinsic 


\begin{tabular}{|c|c|c|c|}
\hline \multicolumn{2}{|c|}{ Nomenclature } & $\aleph$ & the set of all nodes in the mesh \\
\hline \multirow[t]{2}{*}{$\mathbf{a}_{J}$} & nodal enriched degrees of freedom vector associated & $\aleph_{j u n}$ & the set of the nodes whose supports contain a junction \\
\hline & with the Heaviside function & $\aleph_{t i p}$ & the set of the nodes whose supports contain a tip \\
\hline \multirow[t]{2}{*}{$\mathbf{b}_{a K}$} & nodal enriched degrees of freedom vector associated & $\bar{p}$ & the prescribed pressure on the boundary \\
\hline & with Branch functions & $\bar{p}_{m}$ & Pore pressure \\
\hline B & strain operator & $\mathbf{P}$ & pore pressure vector \\
\hline$B_{l}(\mathbf{x})$ & near-tip function & $\bar{q}$ & the volumetric flux on the boundary \\
\hline \multirow[t]{2}{*}{$\mathbf{c}_{M}$} & nodal enriched degrees of freedom vector associated & $R_{p}$ & fracture penetration ratio \\
\hline & with the Junction function & $t$ & time \\
\hline $\mathbf{D}_{\mathbf{e}}$ & elastic matrix & $\overline{\mathbf{t}}$ & the prescribed force on the boundary \\
\hline E & Young modulus & $\Delta t_{n}$ & time step for computation \\
\hline $\mathbf{f}_{u}$ & force vector of displacement & $\mathbf{u}$ & displacement vector of single point \\
\hline $\mathbf{f}_{p}$ & force vector of pressure & $\mathbf{U}$ & displacement vector \\
\hline H & permeability matrix & $\mathbf{w}, \mathbf{w}^{*}$ & the weighting functions \\
\hline$H(\mathbf{x})$ & Heaviside function & $\mathbf{x}$ & the coordinate of domain \\
\hline I & unit tensor & $\alpha$ & declined angle of single crack \\
\hline$I$ & serial number for the standard nodes & $\alpha_{m}$ & Biot coefficient \\
\hline$J$ & serial number for the Heaviside enriched nodes & $\Gamma_{p}$ & the prescribed pressure boundary \\
\hline$J(\mathbf{x})$ & Junction function & $\Gamma_{t}$ & the prescribed force boundary \\
\hline k, $k$ & matrix permeability & $\Gamma_{u}$ & the prescribed displacement boundary \\
\hline $\mathbf{L}$ & the differential operator & $\Gamma_{q}$ & the prescribed flux boundary \\
\hline $\mathbf{L}_{\mathbf{c}}$ & the differential operator & $\varepsilon$ & total strain \\
\hline m & unit vector & $\varepsilon_{\mathrm{e}}$ & effective strain \\
\hline $\mathbf{n}$ & normal vector & $\varepsilon_{\mathrm{v}}$ & volumetric strain \\
\hline \multirow[t]{2}{*}{$\mathbf{N}_{u}(\mathbf{x})$} & standard shape function for displacement & $\theta$ & cracks intersection angle \\
\hline & approximation & $\mu$ & fluid viscosity \\
\hline & standard shape function for pressure approximation & $v$ & Poisson's ratio \\
\hline \multirow[t]{2}{*}{$\widehat{N}_{u}(\mathbf{x})$} & enriched shape function for displacement & $\rho$ & fluid density \\
\hline & approximation & $\boldsymbol{\sigma}$ & matrix stress \\
\hline$\widehat{N}_{p}(\mathbf{x})$ & enriched shape function for pressure approximation & $\varphi$ & matrix porosity \\
\hline$n_{c r}$ & number of fractures & $\Omega$ & computational domain \\
\hline
\end{tabular}

XFEM [18] and enriched meshfree methods [19]. A review of the treatment of cracks by partition of unity methods is provided in [20]. Partition of unity enrichment enables arbitrary functions to be added to the approximation space, e.g. functions which are discontinuous through the crack faces, or singular functions to represent the near-tip behavior. The major advantage of such partition of unity enrichment schemes is that discontinuities can be represented within finite elements, or meshfree domains of influence, thereby avoiding re-meshing. The possible addition of singularities also enables the use of coarser elements in the vicinity of the crack tip.

FEM with interface elements (IEs) are widely used in geotechnical engineering, e.g. specially designed elements such as Goodman's joint elements [21] may be used to represent a fracture. The IEs are used widely to tackle fracture mechanics problems but also hydro-mechanical problem with preexisting fractures $[22,23]$. Recently, Nick et al. [24] proposed a hybrid finite-element finitevolume method to simulate solute transport in discrete fractures and a surrounding porous matrix. They also compared three discretization schemes suitable for discrete fracture modeling [24].

Another fracture modeling method, lattice models, typically used to simulate fractures in cement and other random materials [25], was recently developed to describe the fluid flow in concrete with meso-scale cracks [26]. The advantage of this method is its capacity of heterogeneous simulation.

Herein, we propose to follow the route of partition of unity enrichment, where discontinuities, such as natural and artificial fractures, faults, and interfaces between rock layers, can be represented without requiring the mesh to conform to the, potentially complex, geometry of these evolving discontinuities. Recently, the enriched approximations were used to model flow within a fractured porous medium. Réthoré et al. [27,28] first proposed a two-scale numerical model to simulate fluid flow in fractured, deforming porous media. In this work, the discrete fractures open and grow independently. Watanabe et al. [29] developed a numerical scheme combining XFEM with lower-dimensional interface elements to solve coupled hydro-mechanical problems in discretely fractured porous media. However, in this approach, the lower-dimensional elements have to be located on the edge of solid continuum elements. Lamb et al. developed a numerical scheme integrating a fracture mapping technique with XFEM to capture the fluid flow and rock deformation in the fractured porous media [30]. Huang et al. [31] proposed a new enrichment scheme to model weak discontinuities across a fracture. In these models, the pore pressure of the rock matrix is treated as a weakly discontinuous field, which means the local pressure across fractures is continuous but the pressure gradient is discontinuous. Refer to [32,33] for details on strong and weak discontinuities. However, for sealed fractures, the pressure across cemented cracks is itself discontinuous and hence, strong discontinuities must be incorporated in the approximation.

In this paper, we propose a numerical framework to solve the coupled hydro-mechanical problem associated with flow in cracked porous media, as well as represent the effect of the geologically important sealed fractures. We develop a partition of unity enrichment technique where the pressure field is allowed to be discontinuous across sealed fractures. We also propose a simple approach to handle branching cracks as well as multiple junctions where several cracks meet at a single point. Numerical examples are used to verify the appropriateness of the discretization space to solve the coupled problem. The paper also presents a parametric study on the effects of sealed fractures on fluid flow in porous media. The remainder of this manuscript is outlined as follows. First, we define the problem and summarize the governing equations in 
Section 2.1. The fracture representation and discretization scheme are then presented in Sections 2.2 and 2.3. Numerical experiments designed to validate the proposed framework, flow cases with a single fracture and intersecting fractures, are presented in Section 2.4. Finally, the results of the numerical experiments are presented and discussed in Sections 3 and 4 respectively.

\section{Methodology}

In this paper, sealed fractures are modeled as impermeable interfaces with a certain length and orientation. This is believed to be a reasonable assumption for the practical application we are considering, i.e. the simulation of oil and gas reservoirs [6]. Based on this simple model, we have designed two sets of numerical experiments to study the effect of sealed fractures on fluid behavior, in which fracture length, orientation, and connectivity were set as key parameters. For fractured porous media, stress sensitivity needs to be considered because of the deformability of the rock matrix. To do so, we rely on Biot's consolidation theory [34] and formulate the resulting coupled hydro-mechanical problem. The displacement and pore pressure are both assumed discontinuous across the cracks. We assume transient Darcy flow for the two-phase material (rock and fluid). However, the presented numerical scheme can be also used for non-Darcy flow problems, where the only modification will be the discretization of the diffusion term in the equations presented in the following section.

\subsection{Governing equations}

It is assumed that the deformable porous media is such that the transient fluid flow can be considered "slow", and that the medium is in quasi-static equilibrium. Assuming small displacements and linear elasticity for the rock matrix, the equilibrium equations, kinematics, and the constitutive law of the solid phase without the gravity term can be written, respectively:

$\nabla \times\left(\boldsymbol{\sigma}-\alpha_{m} \bar{p}_{m} \mathbf{I}\right)=0$

$\boldsymbol{\varepsilon}=\frac{1}{2}\left(\nabla \mathbf{u}+(\nabla \mathbf{u})^{T}\right)$

$d \boldsymbol{\sigma}=\mathbf{D}_{\mathrm{e}} d \boldsymbol{\varepsilon}_{\mathrm{e}}$

where $\boldsymbol{\sigma}$ is the effective stress tensor, $\alpha_{m}$ is the Biot coefficient of the porous medium, $\bar{p}_{m}$ is the average fluid pressure in the porous medium. I is the identity tensor, $\mathbf{u}$ is the displacement of the rock matrix, and $\mathbf{D}_{\mathbf{e}}$ is the fourth-order elastic material tensor for plane strain conditions. The kinematics are given by Eq. (2) under the small strain assumptions. Because the hydro-mechanical coupling is nonlinear [35], the constitutive law is given in an incremental form (Eq. (3)).

For subsurface fluid (oil/water) flow in deformable porous media, the mass conservation and Darcy law for transient flow are respectively given by

$\frac{\partial(\rho \varphi)}{\partial t}+\nabla \times(\rho \mathbf{u})=0$

$\mathbf{u}=-\frac{\mathbf{k}}{\mu} \nabla p$

where $\rho, \mu$ denote the density and viscosity of the subsurface fluid respectively, which are assumed constant. $\varphi$ is the porosity of the porous medium. $\mathbf{k}$ is the permeability tensor of the porous medium. To simplify calculations, the porous medium is assumed here to be isotropic; therefore, the permeability tensor degenerates to a diagonal tensor in $2 \mathrm{D}$.
We ignore any change in grain volume, so that the change in volumetric strain is equal to the change in pore volume. Within this assumption, the governing equation for the porous flow can be written as:

$\frac{k}{\mu} \nabla^{2} p-\frac{\partial \boldsymbol{\varepsilon}_{\mathbf{v}}}{\partial t}=0$

where $\boldsymbol{\varepsilon}_{\mathbf{v}}$ denotes the volumetric strain of the rock matrix, which couples the changes in pore pressure and the deformation of the rock.

As shown in Fig. 1, boundary conditions only consist of the displacement and traction on the edge of computational domain, which is given by

$\mathbf{u}=\overline{\mathbf{u}}$ on $\Gamma_{u}$

$\boldsymbol{\sigma} \times \mathbf{n}=\overline{\mathbf{t}} \quad$ on $\quad \Gamma_{t}$

where $\overline{\mathbf{u}}$ represents the prescribed displacement on boundary $\Gamma_{u}$, $\boldsymbol{\sigma}$ is the total stress i.e. the sum of the effective stress and the fluid pressure, and $\overline{\mathbf{t}}$ is the traction vector on boundary $\Gamma_{t}$. Specially, the normal and shear displacements on the crack surfaces must be constrained in order to ensure the proper transfer of forces when the cracks are closed (illustrated in Fig. 1).

Boundary conditions for the fluid flow in the porous medium write:

$p=\bar{p} \quad$ on $\quad \Gamma_{p}$

$-\frac{\mathbf{k}}{\mu}(\nabla p)^{T} \times \mathbf{n}=\bar{q} \quad$ on $\quad \Gamma_{q}$

where $\bar{p}$ is the prescribed pressure on the boundary $\Gamma_{p}$, and $\bar{q}$ is the volumetric flux on the boundary $\Gamma_{q}$.

The initial conditions can be given by the initial values of fluid pressure and displacement. We assume that the pressure reaches equilibrium at the initial time, in which no pressure gradient is present anywhere.

\subsection{Extended finite element approximation of the pressure and displacement fields}

The sealed fractures are modeled as open zero thickness lines. The pressure and displacement are both discontinuous across fractures, because of the impermeability of the materials, which cement the cracks. The enrichment schemes for a single fracture and multiple crossing fractures are introduced separately.

In partition of unity (PU) enrichment frameworks such as the extended finite element method (XFEM), as described in the introduction, discontinuities need not be explicitly meshed, i.e. nodes and element edges need not be placed along the crack faces. Consequently, the discontinuity surfaces may be arbitrarily orientated relative to the mesh, and their position has to be tracked in time. One possibility which has been widely adopted within PUbased methods is to use the level set method [36], where the evolving interfaces are followed implicitly by updating nodal



Fig. 1. Porous domain with cemented cracks and its boundary conditions. 
values of a distance field to the interfaces. The level set method can be used directly for closed interfaces, as it provides distance field to the interface at each time step of a simulation, but must be modified to handle open interfaces such as cracks, in order to be able to track the fronts. We chose to represent the interface by two level set functions as first proposed in [37].

\subsubsection{Single sealed fracture}

We use two level set functions to describe the crack faces; zero and tangential level sets. For details, the reader is referred to [37] for the two-dimensional case, and to [38] for the three dimensional case. From these level set functions, we define a crack coordinate system at each tip in order to compute enrichment functions and we use the level set values to detect enriched nodes: nodes whose supports are split by the crack are enriched with the Heaviside function (discontinuous), and nodes whose supports contain the tip are enriched with near-tip functions. The Heaviside function takes the value +1 on one side of the crack and -1 on the other, as described in detail in [37]. For a review on the treatment of cracks by level sets, the reader is referred to the comprehensive paper of [39]. The level sets can represent the crack location only through two functions, which makes the crack without necessary to align with the background mesh. With this advantage, our model becomes more capable to handle the complicated geometry of cracks, e.g. the curved crack and intersected cracks.

Based on these two sets of enrichment functions, the displacement and pressure approximations for a single fracture can be constructed as

$$
\begin{aligned}
\mathbf{u}^{h}(\mathbf{x})= & \sum_{I \in \aleph} N_{u}^{I}(\mathbf{x}) \mathbf{u}_{I}+\sum_{J \in \aleph_{c r}} \widehat{N}_{u}^{J}(\mathbf{x})\left[H(\mathbf{x})-H\left(\mathbf{x}_{J}\right)\right] \mathbf{a}_{J} \\
& +\sum_{K \in \aleph_{t i p}} \widehat{N}_{u}^{K}(\mathbf{x}) \sum_{l=1}^{4}\left[B_{l}(\mathbf{x})-B_{l}\left(\mathbf{x}_{K}\right)\right] \mathbf{b}_{l K}
\end{aligned}
$$

$p^{h}(\mathbf{x})=\sum_{I \in \aleph} N_{p}^{I}(\mathbf{x}) p_{I}+\sum_{J \in \aleph_{c r}} \widehat{N}_{p}^{J}(\mathbf{x})\left[H(\mathbf{x})-H\left(\mathbf{x}_{J}\right)\right] \mathbf{a}_{J}$

where $\mathrm{u}_{I}, p_{I}$ are the nodal displacement and pressure vectors respectively. $H$ is the Heaviside function and $B_{l}$ are the near-tip functions which span the first order term of the linear elastic asymptotic fields for a crack in an infinite medium. $\aleph$ is the set of all nodes in the mesh, $\boldsymbol{\aleph}_{c r}$ is the set of nodes whose supports are cut by a fracture, and $\aleph_{\text {tip }}$ is the set of the nodes whose supports contain a tip. $a_{J}$ are the nodal enriched degrees of freedom vector associated with the Heaviside function; and $b_{I K}$ are the nodal enriched degrees of freedom vector associated with Branch functions. Both $\mathrm{a}_{J}$ and $\mathrm{b}_{I K}$ are additional degrees of freedom associated with enriched nodes.

The first term of the approximation in Eq. (11) is the standard finite element approximation and is continuous over the whole domain. The second term represents the discontinuity of the displacement field across cracks, and the third term represents the near tip asymptotic displacement field for tip nodes. Note that the main point of partition of unity methods is that the enrichment functions, added to the approximation field, can be reproduced exactly, locally in the computational domain, without requiring any global enrichment strategy, nor introducing special elements such as Barsoum elements [40]. Additionally, the enrichment functions were specially designed according to the characters of the sealed fracture. First, the sealed fracture belongs to the strong discontinuity that the displacement and pressure across the crack exists a jump, so the Heaviside function becomes the good candidate to capture this feature. Second, the asymptotic behavior of displacement near the tips of sealed fracture agrees that the displacement value is proportional to the square root of the distance to the tip in the frame of linear fracture mechanics.

\subsubsection{Multiple crossing fractures}

To represent intersecting fractures, we follow [41], where a primary fracture is defined along with secondary fractures joining the primary fracture. Elements that contain a junction, i.e. the intersection of at least two fractures are enriched with a junction function. Junction nodes are only activated for secondary cracks, not for the primary crack. An alternative, systematic and simple approach was proposed by $[42,43]$ in the context of polycrystalline fracture.

The value of the junction function depends on the crossing condition between the main fracture and the branching fractures. As shown in Fig. 2, a local Cartesian coordinate system for the main crack is established, in which the main fracture (bold line) is aligned with axis $\xi_{\Gamma}$. We consider two crossing conditions, which are characterized by the intersection angle, $\theta$. Counter-clockwise angles are taken as positive. Then the junction function can be written as:

(I) if $0^{\circ}<\theta<180$

$$
J(\mathbf{x})=\left\{\begin{array}{cc}
H_{2}(\mathbf{x}) & \text { for } H_{1}(\mathbf{x})<0 \\
0 & \text { for } H_{1}(\mathbf{x})>0
\end{array}\right.
$$

(II) if $180^{\circ}<\theta<360$

$$
J(\mathbf{x})=\left\{\begin{array}{cc}
H_{2}(\mathbf{x}) & \text { for } H_{1}(\mathbf{x})>0 \\
0 & \text { for } H_{1}(\mathbf{x})<0
\end{array}\right.
$$

where $H_{1}$ is the Heaviside function of the main fracture, and $H_{2}$ is the Heaviside function of the branch. The proposed junction function presented in this paper is an adaptation of the methodology developed by Daux et al. [41].

Let $n_{c r}$ be the number of fractures (the main fracture plus the number of secondary fractures), $\aleph_{j u n}$ be the set of the nodes whose supports contain a junction. The approximations for the displacement field and the pressure field are now written as

$$
\begin{aligned}
\mathbf{u}^{h}(\mathbf{x})= & \sum_{I \in \aleph} N_{u}^{I}(\mathbf{x}) \mathbf{u}_{I}+\sum_{J \in \aleph_{c r}} \widehat{N}_{u}^{J}(\mathbf{x}) \mathbf{a}_{J} \sum_{i=1}^{n_{c r}}\left[H(\mathbf{x})-H\left(\mathbf{x}_{J}\right)\right] \\
& +\sum_{K \in \aleph_{\text {tip }}} \widehat{N}_{u}^{K}(\mathbf{x}) \sum_{i=1}^{n_{c r}} \sum_{l=1}^{4}\left[B_{l}(\mathbf{x})-B_{l}\left(\mathbf{x}_{K i}\right)\right] \mathbf{b}_{l K i} \\
& +\sum_{M \in \aleph_{j u n}} \widehat{N}_{u}^{M}(\mathbf{x}) \mathbf{c}_{M}\left[J(\mathbf{x})-J\left(\mathbf{x}_{M}\right)\right] \\
p^{h}(\mathbf{x})= & \sum_{I \in \aleph} N_{p}^{I}(\mathbf{x}) p_{I}+\sum_{J \in \aleph_{c r}} \widehat{N}_{u}^{J}(\mathbf{x}) \mathbf{a}_{J} \sum_{i=1}^{n_{c r}}\left[H(\mathbf{x})-H\left(\mathbf{x}_{J}\right)\right] \\
& +\sum_{M \in \aleph_{j u n}} \widehat{N}_{u}^{M}(\mathbf{x}) \mathbf{c}_{M}\left[J(\mathbf{x})-J\left(\mathbf{x}_{M}\right)\right]
\end{aligned}
$$

in which the subscript $i$ refers to the ID of the $i$ th fracture.



Fig. 2. Locally Cartesian coordinate system for fractures. 


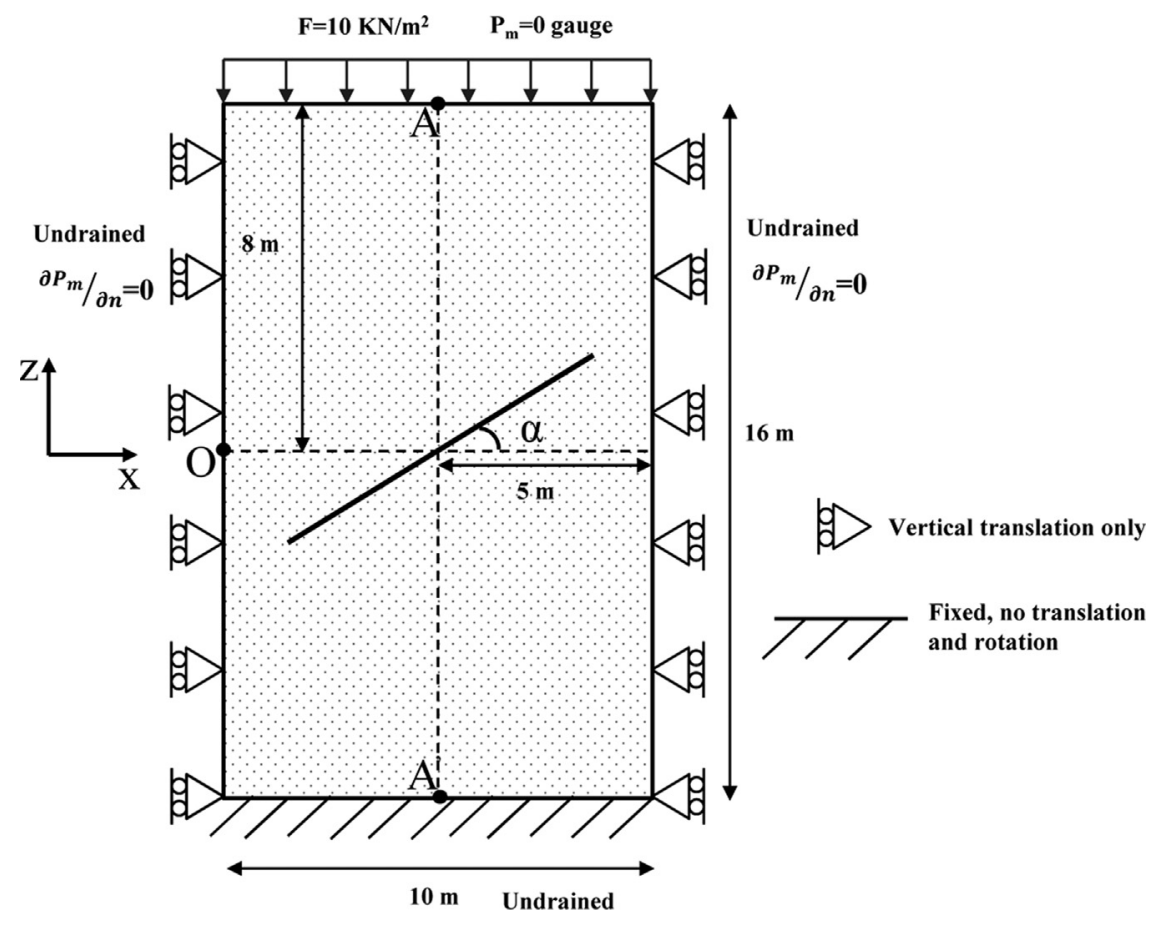

Fig. 3. Schemetic of single fracture model.

2.3. Weak form and semi-discrete finite element equations from the weighted residual method

Using the weighted residual method the weak form of the governing equations are derived. Multiplying the equilibrium equation (Eq. 1) by a weight function, and integrating over the domain provides the following equation:

$\int_{\Omega}(\mathbf{L w})^{T} \boldsymbol{\sigma}(\mathbf{u}, p) d \Omega-\int_{\Gamma_{\mathbf{t}}} \mathbf{w}^{T} \overline{\mathbf{t}}(\mathbf{u}, p) d \Gamma=\mathbf{0}$

where $\mathbf{L}$ is the symmetric gradient differential operator, and $\mathbf{w}$ is a weight function.

The weighted residual method is also applied to the mass balance equation for fluid flow. The weak form can be written as

$\int_{\Omega}\left[\left(\nabla \mathbf{w}^{*}(\mathbf{u}, p)\right)^{T}\left(-\frac{\mathbf{k}}{\mu} \nabla p\right)+\mathbf{w}^{* T}(\mathbf{u}, p) \alpha_{m} \mathbf{m}^{T} \mathbf{L} \frac{\partial \mathbf{u}}{\partial t}\right] d \Omega+\int_{\Gamma_{q}} \mathbf{w}^{* T}(\mathbf{u}, p) \bar{q} d \Gamma=0$

where $\mathbf{w}^{*}$ is a weight function. The primary unknowns are the displacement $\mathbf{u}$ and flow pressure $p$ in the porous medium.

In the extended finite element method proposed here, we follow a Bubnov-Galerkin approach and choose the weighting functions $\mathbf{w}$ and $\mathbf{w}^{*}$ as the usual finite element shape functions, augmented with appropriate enrichment functions. According to the enrichment schemes for displacement and pressure described in Section 2.2, we use the regular displacement degrees of freedom $\mathbf{u}_{I}$, the enriched degrees of freedom $\mathbf{a}_{j}$ and $\mathbf{b}_{J}$, and the pressure $p$ as primary variables for the discretization. The approximations for $\mathbf{u}$ and $p$ are expressed as:

$\mathbf{u}^{h}(\mathbf{x}, t)=\mathbf{N}_{u}(\mathbf{x}) \mathbf{U}(t)$

$p^{h}(\mathbf{x}, t)=\mathbf{N}_{p}(\mathbf{x}) \mathbf{P}(t)$

where $\mathbf{N}_{u}, \mathbf{N}_{p}$ are the usual Lagrange shape functions. $\mathbf{U}$ and $\mathbf{P}$, are the vectors of the nodal values of the unknowns, which are given as
Table 1

The input parameters for the mentioned model.

\begin{tabular}{llll}
\hline Parameter & Symbol & Value & Unit \\
\hline Initial reservoir pressure & $P_{0}$ & 1000 & $\mathrm{~Pa}$ \\
Young's modulus & $E$ & 40 & $\mathrm{MPa}$ \\
Poisson's ratio & $n$ & 0.3 & No dimension \\
Matrix porosity & $\phi$ & 0.1 & No dimension \\
Matrix fluid mobility & $k / \mu$ & $6 \mathrm{E}-12$ & $\mathrm{~m}^{3} \mathrm{~s} / \mathrm{kg}$ \\
\hline
\end{tabular}

$\mathbf{U}=\left\{\mathbf{u}_{1}, \mathbf{u}_{2}, \mathbf{u}_{3}, \ldots, \mathbf{u}_{N}, \mathbf{a}_{1}, \mathbf{a}_{2}, \mathbf{a}_{3}, \ldots, \mathbf{a}_{N_{c r}}, \mathbf{b}_{1}, \mathbf{b}_{2}, \mathbf{b}_{3}, \ldots, \mathbf{b}_{N_{\text {tip }}}\right\}^{T}$

$\mathbf{P}=\left\{\mathbf{u}_{1}, \mathbf{u}_{2}, \mathbf{u}_{3}, \ldots, \mathbf{u}_{N}, \mathbf{a}_{1}, \mathbf{a}_{2}, \mathbf{a}_{3}, \ldots, \mathbf{a}_{N_{c r}}\right\}^{T}$

The weak forms of the governing equations then write:

$\int_{\Omega}\left(\mathbf{L N}_{u}\right)^{T} \boldsymbol{\sigma} d \Omega-\int_{\Gamma_{t}} \mathbf{N}_{u}^{T} \overline{\mathbf{t}} d \Gamma=0$

$\int_{\Omega}\left(\nabla \mathbf{N}_{p}\right)^{T} \frac{\mathbf{k}}{\mu} \nabla \mathbf{N}_{p} \mathbf{P} d \Omega+\int_{\Omega} \mathbf{N}_{p}^{T} \alpha \mathbf{m}^{T} \mathbf{L} \mathbf{N}_{u} \frac{\partial \mathbf{U}}{\partial t} d \Omega+\int_{\Gamma_{q}} \mathbf{N}_{p}^{T} \bar{q} d \Gamma=0$

Taking into account the effective stress, Eqs. (22) and (23) are rewritten as:

$\int_{\Omega} \mathbf{B}^{T} \boldsymbol{\sigma}^{\prime} d \Omega-\mathbf{L}_{c} \mathbf{P}=\mathbf{f}_{\mathbf{u}}$

$\mathbf{H P}+\mathbf{L}_{c}^{T} \frac{d \mathbf{U}}{d t}=\mathbf{f}_{p}$

To treat the nonlinear problem, Eq. (24) is given in incremental form:

$\mathbf{K} \frac{d \mathbf{U}}{d t}+\mathbf{L}_{c} \frac{d \mathbf{P}}{d t}=\frac{d \mathbf{f}_{u}}{d t}$

where,

$\mathbf{K}=-\int_{\Omega} \mathbf{B}_{\mathbf{u}}^{T} \mathbf{D B}_{\mathbf{u}} d \Omega$ 
$\mathbf{L}_{c}=-\int_{\Omega} \mathbf{B}_{\mathbf{u}}^{T} \mathbf{m} \mathbf{N}_{p} d \Omega$

$d \mathbf{f}_{\mathbf{u}}=-\int_{\Gamma_{t}} \mathbf{N}_{\mathbf{u}}^{T} d \overline{\mathbf{t}} d \Gamma$
$\mathbf{H}=\int_{\Omega}\left(\nabla \mathbf{N}_{p}\right)^{T} \frac{\mathbf{k}}{\mu} \nabla \mathbf{N}_{p} d \Omega$

in which, $\mathbf{B}_{\mathbf{u}}$ is the strain matrix operator. Gauss Legendre quadrature was used for integration. Note that, due to the presence of non-polynomial functions of discontinuities and



Fig. 4. Intersecting fracture pattern (a) branched crack (b) three fractures intersecting at one point (c) arrays of fractures.

a

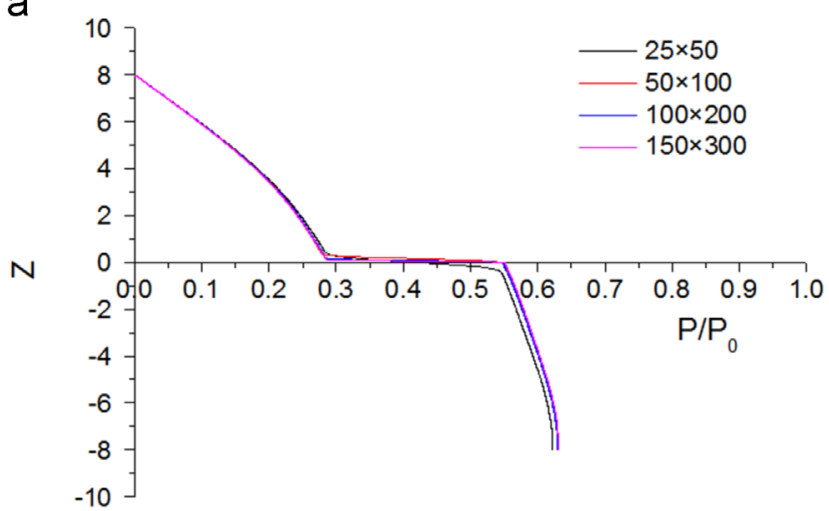

b

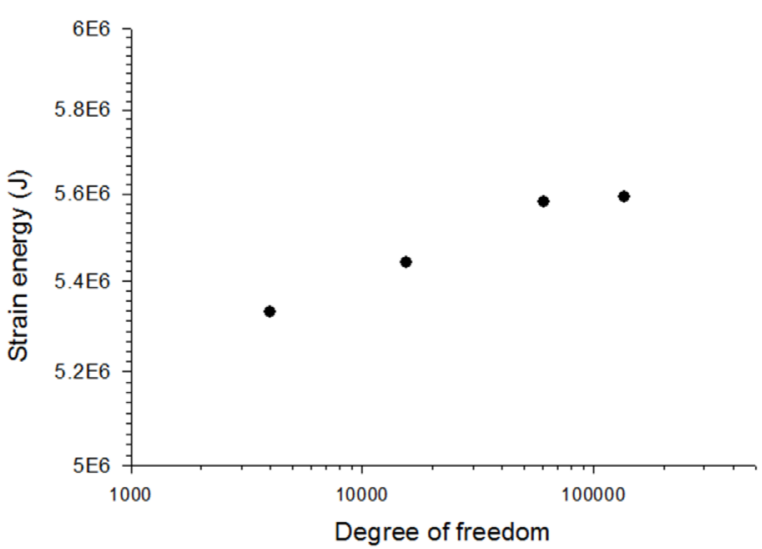

C

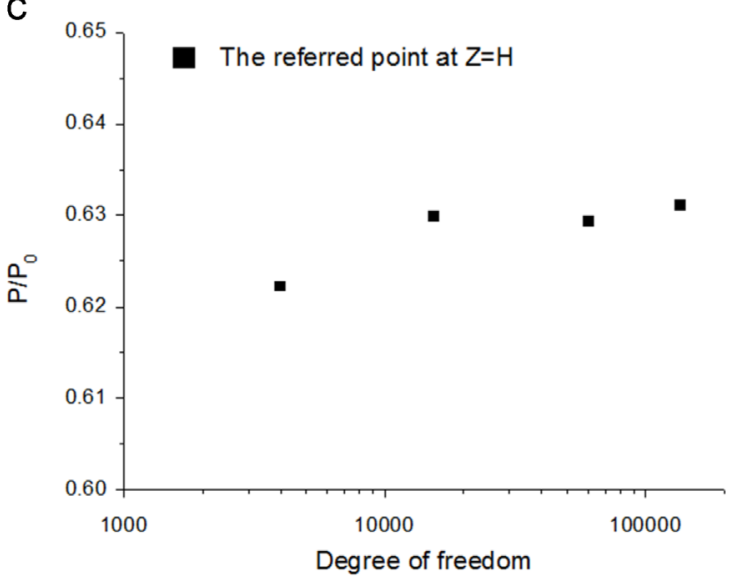


(c) the strain energy of the whole domain. 

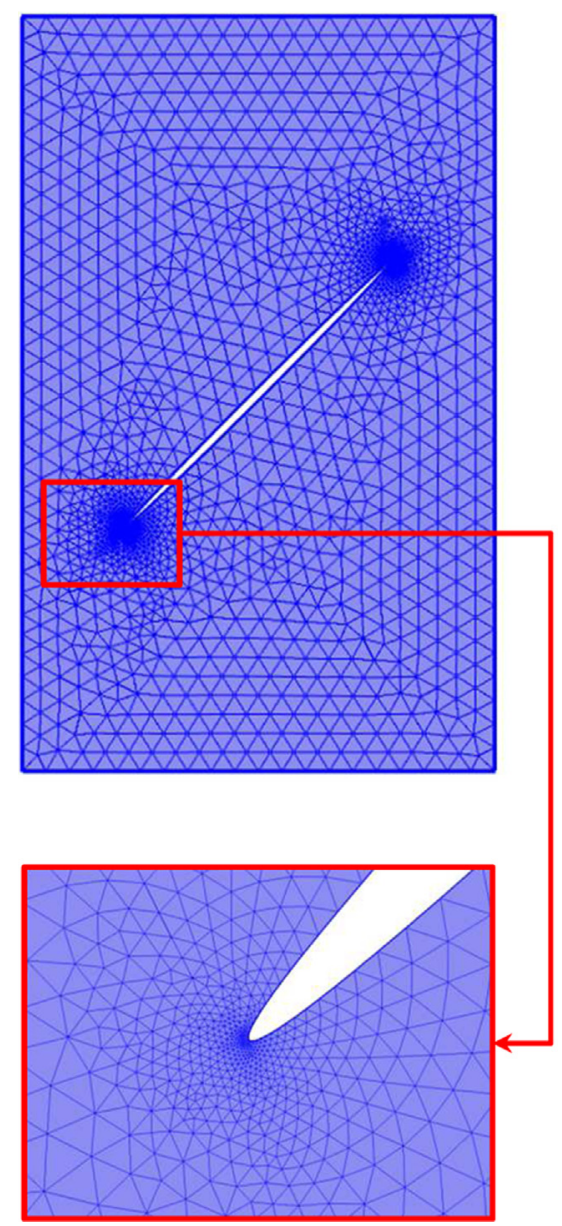

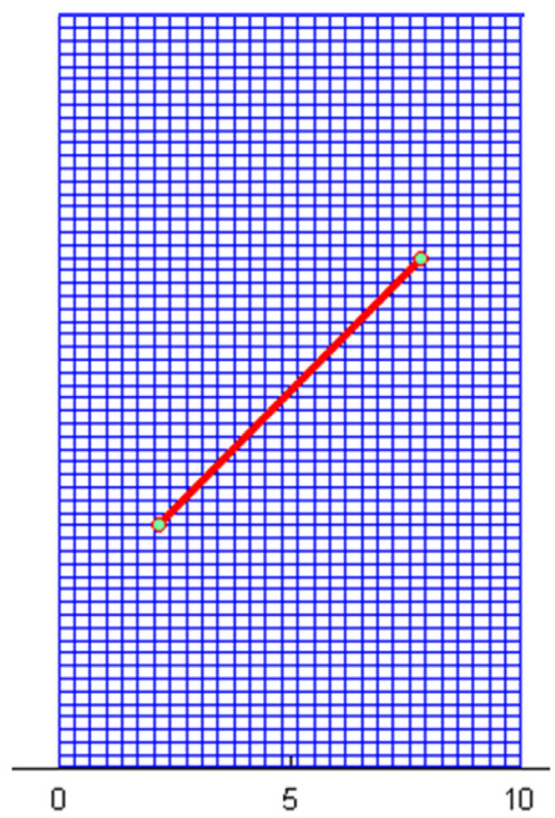

10

Fig. 6. Computational meshes for the SFEM (left) and XFEM (right).

singularities in the approximations, special integration techniques are necessary for enriched approximations. A recent review of such techniques is given in [44].

The temporal discretization is achieved using the generalized trapezoidal method, which is an implicit method. A field function $\mathbf{U}$ can be evaluated in the time interval $\left[t_{n}, t_{n+1}\right]$ :

$\mathbf{U}^{n+\alpha_{t}}=\left(1-\alpha_{t}\right) \mathbf{U}^{n}+\alpha_{t} \mathbf{U}^{n+1}$

where $\alpha_{t}=\left(t-t_{n} / \Delta t_{n}\right) \quad\left(0 \leq \alpha_{t} \leq 1\right)$

Once integrated within a single set of matrix equations, the spatial and the implicit matrix equations are derived as:

$$
\left[\begin{array}{cc}
\mathbf{K} & \mathbf{L}_{c} \\
\mathbf{L}_{c}^{T} & \mathbf{H} \Delta t_{n} \alpha_{t}
\end{array}\right]\left[\begin{array}{l}
\mathbf{U} \\
\mathbf{P}
\end{array}\right]_{t_{n+1}}=\left[\begin{array}{cc}
\mathbf{K} & \mathbf{L}_{c} \\
\mathbf{L}_{c}^{T} & \mathbf{H} \Delta t_{n}\left(1-\alpha_{t}\right)
\end{array}\right]\left[\begin{array}{l}
\mathbf{U} \\
\mathbf{P}
\end{array}\right]_{t_{n}}+\Delta t_{n}\left[\begin{array}{c}
\frac{d \mathbf{f}_{\mathbf{u}}}{d t} \\
\mathbf{f}_{p}
\end{array}\right]
$$

The above formulation was coded using XFEM with 4-node quadrilateral elements in MATLAB based on the open-source codes available here ${ }^{2}$, and detailed in [45]. Further, the same order shape functions were used for both the pressure and displacement approximations. The time step was chosen as $86,400 \mathrm{~s}$ ( 1 day). The numerical experiments designed to study the effect of sealed fractures on flow in porous media are presented in the following section.

\footnotetext{
${ }^{2}$ http://sourceforge.net/users/cmechanicsos, http://sourceforge.net/p/permix/ discussion.
}

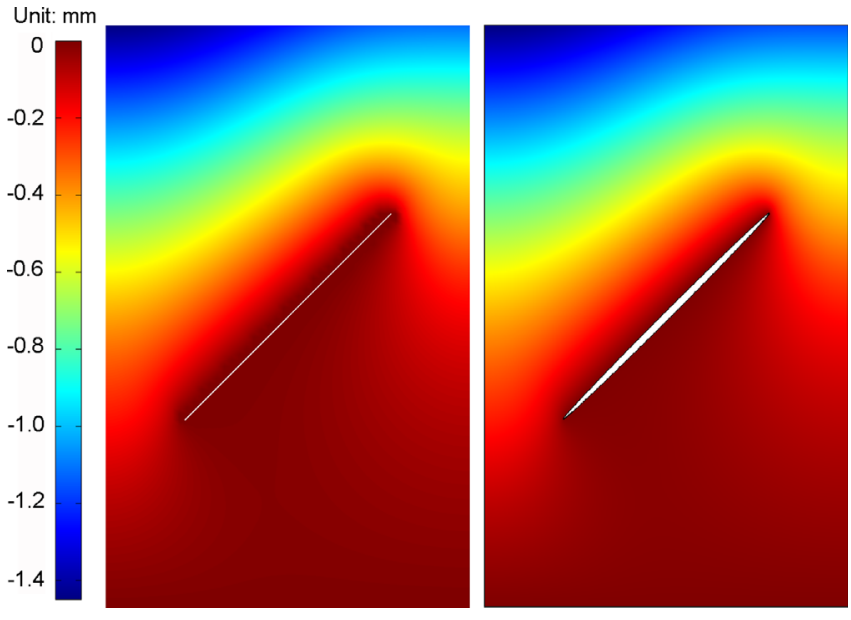

Fig. 7. Displacement fields after 100 days for XFEM (left) and SFEM (right).

\subsection{Crack configurations}

Two problems with both a single fracture and multiple intersecting fractures were chosen to verify the proposed formulation and study the effect of sealed fractures on flow behavior in porous media. To the authors' knowledge, no theoretical displacement and pressure solutions are available for cases involving discrete fractures. Therefore, we have verified the method by studying the convergence of the pore pressure and strain energy with mesh refinement. The influence of the geometry of a single fracture was also analyzed to study its impact on fluid flow. 


\subsubsection{Single sealed fracture model}

Following Terzaghi's problem [46], a single fracture test case was built to verify the hydro-mechanical coupling and the variation of the pressure field. As shown in Fig. 3, the computational domain is a rectangle of width $10 \mathrm{~m}$ and height $16 \mathrm{~m}$ under plane strain conditions, in which a fracture of length $L$ is embedded at an angle of $\alpha$. The top is permeable, and other boundaries are undrained. The pore pressure on the top edge is set to zero. A uniform constant force of $10 \mathrm{kN} / \mathrm{m}^{2}$ is applied along the top boundary initially and maintained throughout the duration of the simulation. This force will alter the pore pressure distribution and induce a flow field from the bottom to the top.
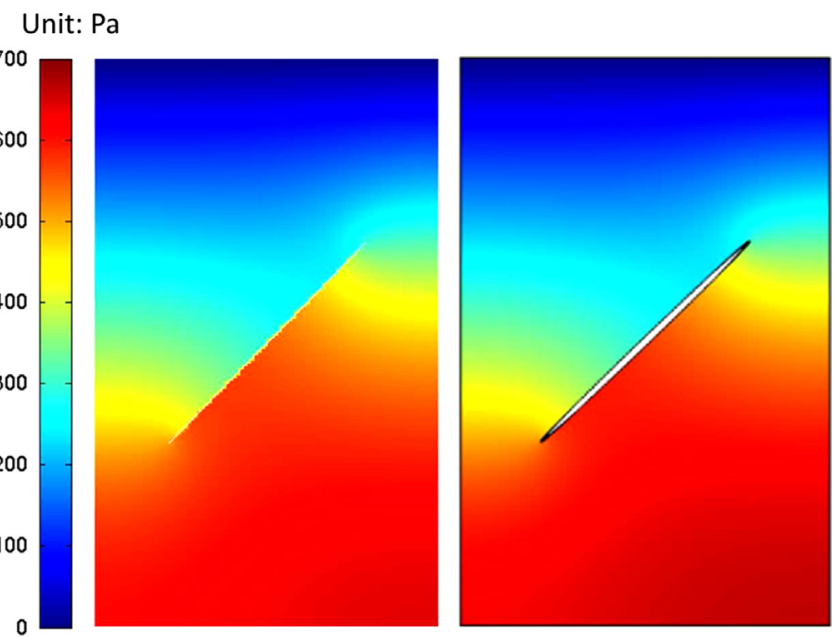

Fig. 8. Pore pressure fields after 100 days for XFEM (left) and SFEM (right).
The left and right boundaries are fixed in the $X$-direction and the bottom boundary is fixed in both the $X$ and the $Y$ directions. Because minerals cement the fracture, the fracture cannot open, and the displacement field should therefore be continuous across the fracture. Besides, the domain is assumed isotropic and to deform linear elastically. The chosen material properties are given in Table 1.

First, four structured meshes with increasing levels of uniform refinement were produced to quantify mesh dependence and convergence with the following mesh densities $25 \times 50,50 \times 100$, $100 \times 200$, and $150 \times 300$. The nodal values of pressure along the $Y$ direction are drawn along the vertical line $A-A^{\prime}\left(A-A^{\prime}\right.$ shown in Fig. 3$)$.

Second, five inclination angles $\left(\alpha=0^{\circ}, 30^{\circ}, 45^{\circ}, 60^{\circ}, 90^{\circ}\right)$ and four fracture lengths $(L=8,6,4,2 \mathrm{~m})$ are considered to show the effect of those parameters on the pressure field. To facilitate the description, we defined the "penetration ratio" for an embedded fracture as the length of fracture divided by the width of the computational domain.

\subsubsection{Multiple crossing fractures model}

Three fracture patterns, including branch intersection, junction intersection, and arrays of fractures, are examined to verify numerically that the proposed model and discretization are able to represent multiple intersecting fractures correctly. The cases considered are shown in Fig. 4. The first two cases have only single intersecting point, but the third one has three intersecting points to simulate arrays of fractures. Boundary conditions are the same as for the single sealed fracture example. Material properties of the porous matrix are listed in Table 1.

Intersecting fractures are treated as a main fracture along with several secondary fractures. In Fig. 4, the solid line represents the main fracture and dotted lines denote secondary fractures.

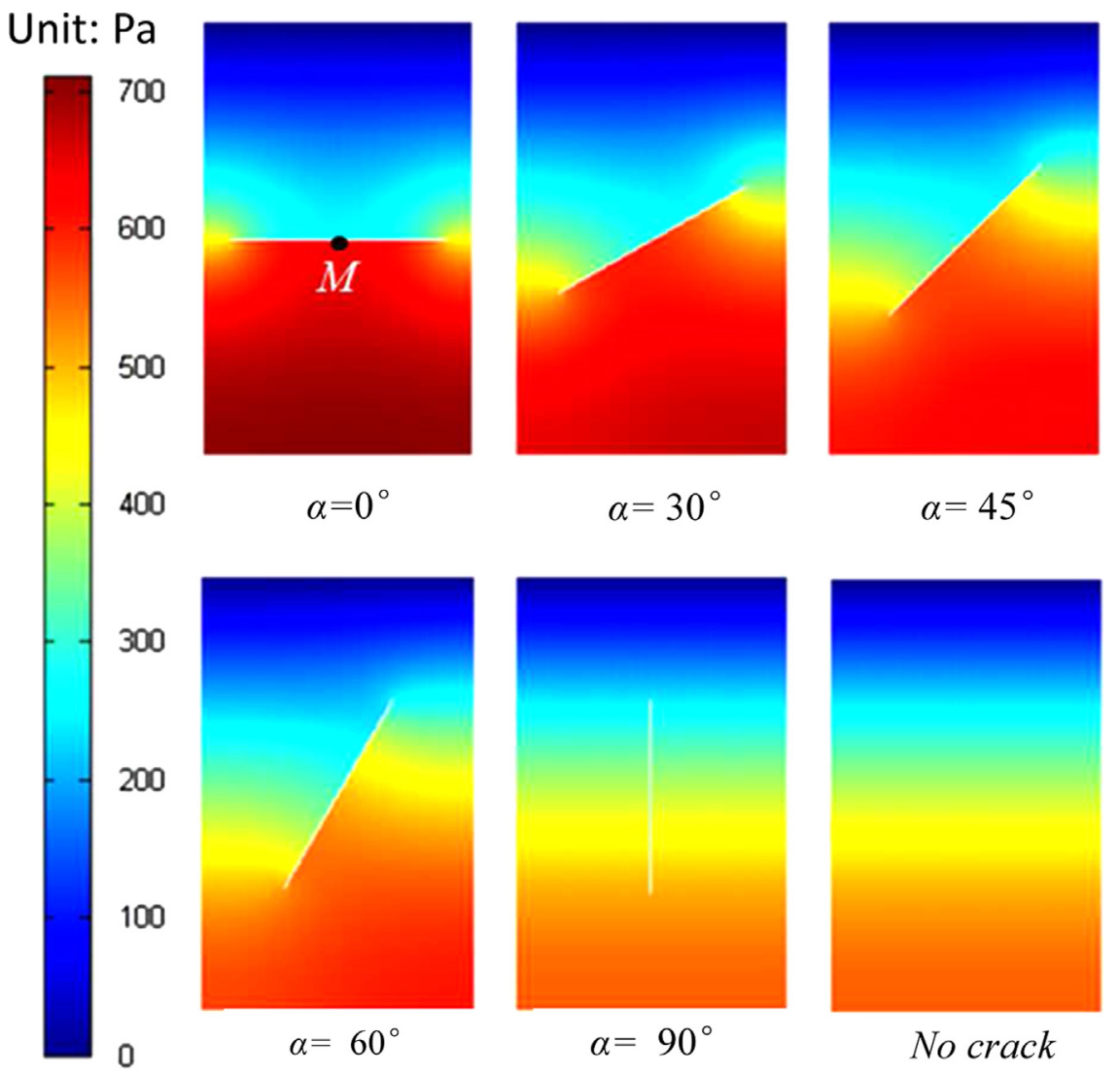

Fig. 9. Pore pressure profiles with different inclination angles. The last one is for comparison without any fracture. 


\section{Results}

In this section, convergence tests are presented to study the appropriateness of the discretization space and verify the implementation. Then, the effects of the inclination angle and length of a single fracture on the pressure field are studied through numerical experiments. Finally, pressure fields for multiple fractures are commented.

\subsection{Single sealed fracture model}

\subsubsection{Verification of the approximation properties and of the implementation}

We define a non-dimensional, normalized pressure field as the ratio between the flow pressure at a given point and time divided by the initial pressure in the domain. The non-dimentional pressure along line $a-a^{\prime}$ is compared for four different mesh sizes in Fig. 5(a). We choose $z=-8 \mathrm{~m}$ as the reference point and show in Fig. 5(b) shows that the strain energy of the domain also converges with mesh refinement. Fig. 5(c) the pore pressure convergence at the reference point. The convergence tests verify the proposed numercial scheme and we can conclude that 20,000 elements are required to obtain $1 \%$ error compared to the converged solution with 60,790 dofs.

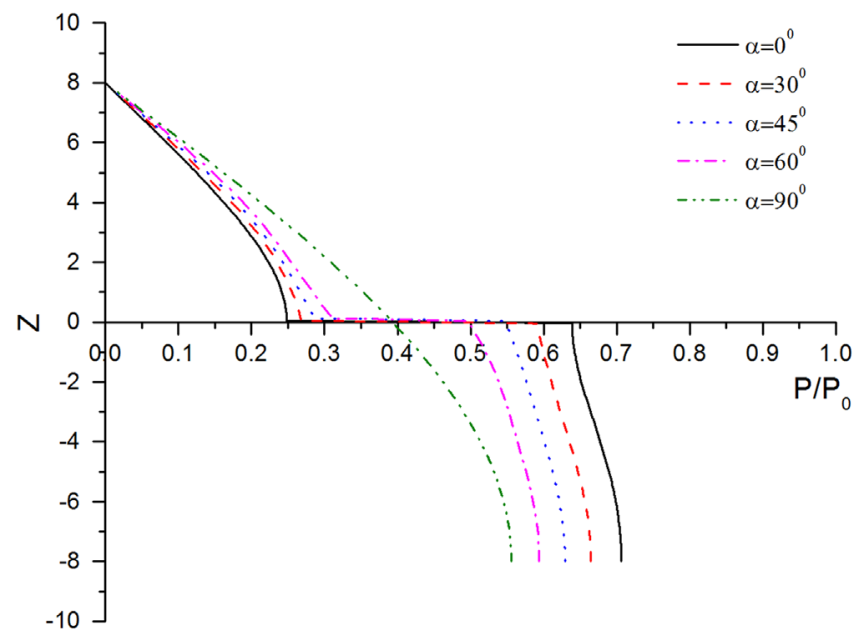

Fig. 10. Non-dimensional pressure profile along line $a-a^{\prime}$ for different inclination angles (shown in Fig. 3). $P_{0}$ denotes the intial pressure and $P$ denotes the pore pressure at the 100th day.

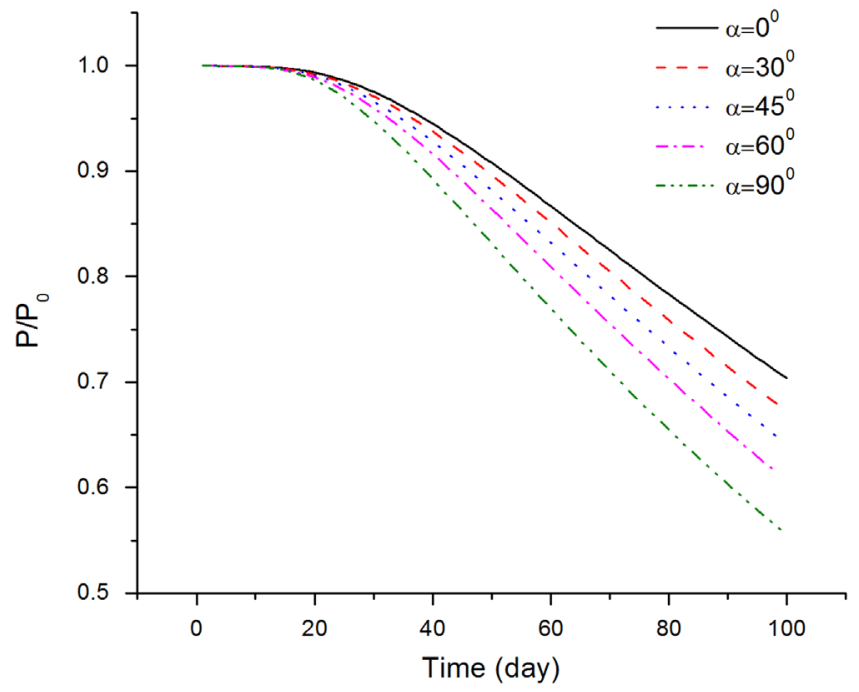

Fig. 11. Time history of the non-dimensional average pressure (defined as the average pressure of field overs the initial pressure ) for different inclination angle.
The presented model is compared with a standard finite element model (SFEM) in which the sealed fracture is modeled as an elliptical void. The void has an aspect ratio of $1: 40$ with the boundary conditions of no flow through its surface and traction free at the surface. The displacements on the void surface were fixed to be zero because of the sealed fracture simulation.

The computational meshes used for both the SFEM and XFEM are shown in Fig. 6. The vicinity of fracture tips in the SFEM mesh are refined by triangular three/six node element with a total of 30,742 elements. A uniform mesh with 20,000 elements was used for the XFEM to demonstrate the method's capability to accurately simulate coupled deformation and flow around a discontinuity.

The plots of displacement fields and pore pressure fields after 100 days are shown in Figs. 7 and 8. The results from both XFEM and SFEM are qualitatively close.

\subsubsection{Effect of inclination angle of the fracture}

Fig. 9 shows the pore pressure profiles for different inclination angles on the 100th day. Fig. 10 shows the pore pressure varies with the vertical coordinates along line $A-A^{\prime}$. As shown in Fig. 9, the pore pressure across a sealed fracture systematically shows a discontinuity, except at angle $90^{\circ}$. This implies that a fracture aligned with the flow

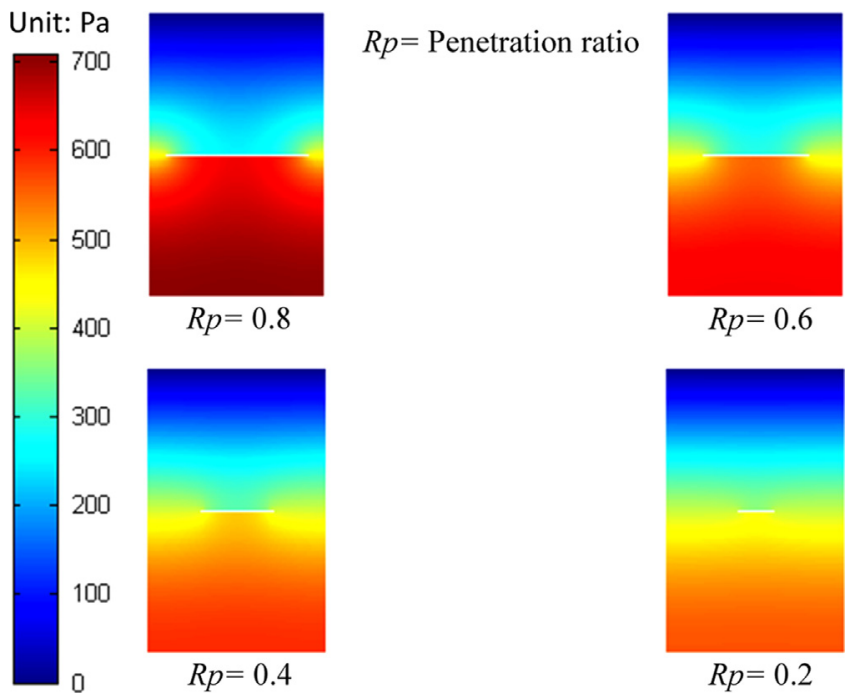

Fig. 12. Pore pressure profiles with different fracture lengths.



Fig. 13. Time history of the non-dimensionally average pressure for different penetration ratios. 
direction does not disturb the pressure field, which conforms to intuition. Besides, the pressure jump increases with decreasing inclination angle and is maximum when the fracture is orthogonal to the flow direction. Furthermore, the rate at which the pore pressure decreases above the midpoint (point $M$ ) increases with decreasing inclination angles. However, the rate of decline below the midpoint is virtually the same whatever the inclination of the crack is. Fig. 11 shows the average pressure decreases with time. It can be found that the rate of decline in pressure with smaller angles is slower compared to that obtained with larger angles. Therefore, except for the vertical fracture, the inclination angle plays an important role to lower the declined rate of pore pressure. The horizontal fracture, which is orthogonal to the flow direction, has the largest influence on the pore pressure field.

\subsubsection{Effect of fracture length}

A horizontal fracture is chosen to study the effect of fracture length on the pore pressure field. Fig. 12 shows the pore pressure profile for different penetration ratios (defined as the fracture length over the horizontal length of the domain). It illustrates that the pressure jump increases with the penetration ratio. There exists a larger pressure gradient around fracture tips, and the pressure field is axially symmetric. As shown in Fig. 13, the rate of decline of the average pressure is larger with decreasing penetration ratios.

\subsection{Intersecting cracks in porous media}

Fig. 14 shows the pore pressure and displacement profiles for an inserted ' $T$ ' configuration. The vertical branch does not influence the flow behavior because there is no pressure jump across it, as in the case for a single vertical fracture. The pressure is however discontinuous across the horizontal fracture. The displacement profile is symmetric in the $Y$-direction and the displacement on the fracture surface is zero.

Fig. 15 shows the pore pressure and displacement profiles for an asterisk crack pattern. The pore pressure is discontinuous across
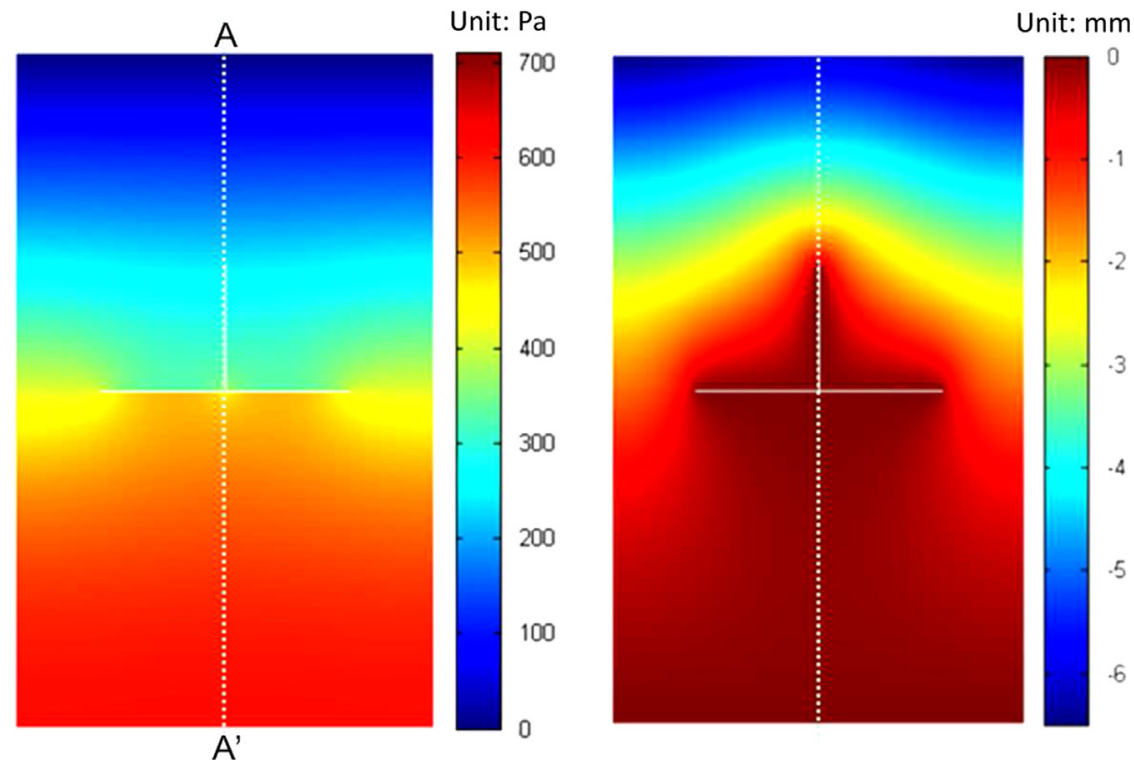

Fig. 14. Inserted ' $T$ ' crack profile, white lines represent cracks (a) Left: Pore pressure profile (b) Right: $Y$ displacement profile.

A

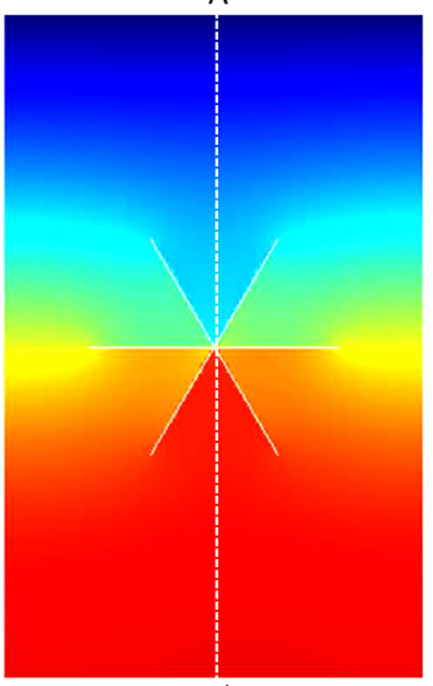

$A^{\prime}$
Unit: $\mathrm{Pa}$



Unit: $\mathrm{mm}$

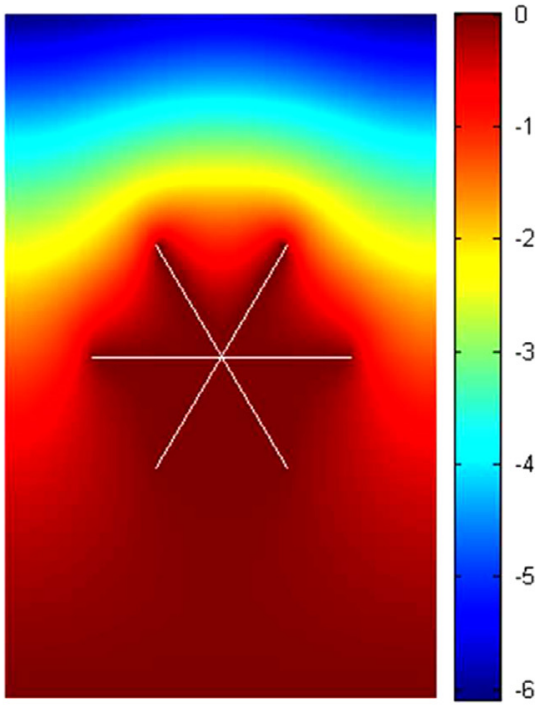

Fig. 15. Asterisk crack pattern, white lines represent fractures (a) Left: Pore pressure profile (b) Right: $Y$ displacement profile. 
A

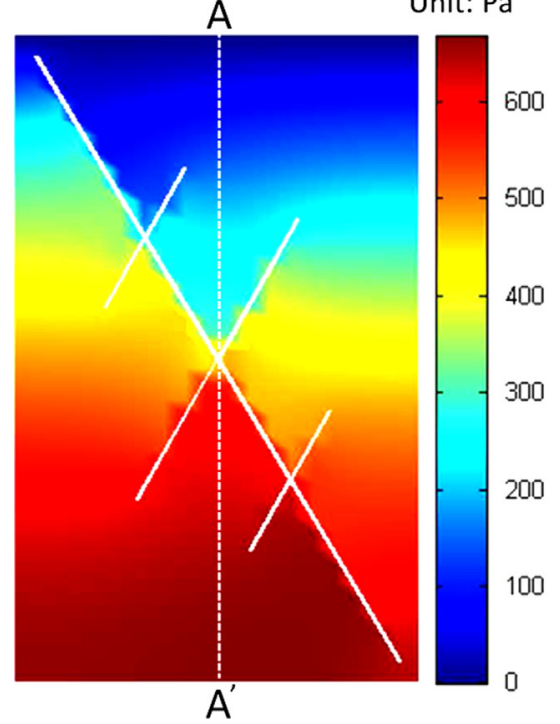

Unit: $\mathrm{mm}$



Fig. 16. Arrays of fractures, white lines represent fractures-(a) left: pore pressure profile; (b) right: $Y$ displacement profile.

the cracks. Since the geometry of the pattern is symmetric with respect to axis $A-A^{\prime}$, the pore pressure and displacement fields are also symmetric.

Fig. 16 shows the pore pressure and displacement profiles for an array of fractures. The pore pressure is discontinuous across the cracks. In this case, we have a long sealed fracture across the domain from top-left to bottom-right, which acts as a barrier to prevent the mass transfer between two sides of the fracture. Therefore, the pressure in the top-right area decreased faster than the bottom-left area.

\section{Discussions and conclusion}

We have presented a numerical framework to simulate coupled deformation and fluid flow in porous media, which also addresses the arbitrary orientation and intersection of sealed fractures. The method can treat such fractures without requiring the mesh to conform to the fractures. Numerical experiments demonstrate the ability of the framework to accurately describe the coupled hydromechanical behavior in a domain containing sealed fractures. The numerical results also confirm that the inclination angle of the fractures with respect to the main fluid flow direction as well as the penetration ratio within the sealed fracture are two factors which significantly affect fluid flow.

The numerical framework presented is currently being extended to encompass fracture propagation and numerical experiments are being developed to determine the method's ability to accurately simulate hydro-mechanical fracturing.

\section{Acknowledgements}

This work was financially supported by the Major International (Regional) Joint Research Program of National Natural Science Foundation of China (No. 51210006). The authors are grateful for approval to publish. Specially, Mao Sheng would like to thank China Scholarship Council for supporting his Joint Advisory PhD Program, and would like to thank the Well Construction Technology Center of the University of Oklahoma for providing him an opportunity to work as a visiting scholar.

\section{References}

[1] Neuman SP. Trends, prospects and challenges in quantifying flow and transport through fractured rocks. Hydrogeol J 2005;13:124-47.

[2] Berkowitz B. Characterizing flow and transport in fractured geological media: a review. Adv Water Resour 2002;25:861-84.

[3] Laubach SE. Practical approaches to identifying sealed and open fractures. AAPG Bull 2003;87:561-79.

[4] Nelson RA. Geologic analysis of naturally fractured reservoirs. Gulf Professional Publishing; 2001.

[5] Apiwathanasorn S, Ehlig-Economides C. Evidence of reopened microfractures in production data analysis of hydraulically fractured shale gas wells, In: SPE Canadian unconventional resources conference 2012.

[6] Gale JFW, Reed RM, Holder J. Natural fractures in the Barnett Shale and their importance for hydraulic fracture treatments. AAPG Bull 2007;91:603-22.

[7] Baraka-Lokmane S, Teutsch G, Main I. Influence of open and sealed fractures on fluid flow and water saturation in sandstone cores using magnetic resonance imaging. Geophys J Int 2001;147:263-71.

[8] Barsoum R, Loomis R, Stewart B. Analysis of through cracks in cylindrical shells by the quarterpoint elements. Int J Fract 1979;15:259-80.

[9] Melenk JM, Babuška I. The partition of unity finite element method: basic theory and applications. Comput Methods Appl Mech Eng 1996;139:289-314.

[10] Babuska I, Melenk JM. The partition of unity finite element method. College Park, MD: Institute for Physical Science and Technology, Maryland University; 1995.

[11] ES NM, Dolbow J, Belytschko T. A finite element method for crack growth without remeshing. Int J Numer Method Eng 1999;46:131-50.

[12] Dolbow J, Moës N, Belytschko T. Discontinuous enrichment in finite elements with a partition of unity method. Finite Elem Anal Des 2000;36:235-60.

[13] Strouboulis T, Babuška I, Copps K. The design and analysis of the generalized finite element method. Comput Methods Appl Mech Eng 2000;181:43-69.

[14] Vu-Bac N, Nguyen-Xuan H, Chen L, Bordas S, Kerfriden P, Simpson R, et al. A node-based smoothed extended finite element method (NS-XFEM) for fracture analysis. Comput Model Eng Sci(CMES) 2011;73:331-55.

[15] Chen L, Rabczuk T, Bordas S, Liu G, Zeng K, Kerfriden P. Extended finite element method with edge-based strain smoothing (ESm-XFEM) for linear elastic crack growth. Comput Methods Appl Mech Eng 2012;209:250-65.

[16] Bordas S, Natarajan S, Kerfriden P, Augarde CE, Mahapatra DR, Rabczuk T, et al. On the performance of strain smoothing for quadratic and enriched finite element approximations (XFEM/GFEM/PUFEM). Int J Numer Methods Eng 2011;86:637-66.

[17] Bordas S, Rabczuk T, Hung N-X, Nguyen VP, Natarajan S, Bog T, et al. Strain smoothing in FEM and XFEM. Comput Struct 2010;88:1419-43.

[18] Fries TP, Belytschko T. The intrinsic XFEM: a method for arbitrary discontinuities without additional unknowns. Int J Numer Methods Eng 2006;68:1358-85.

[19] Fleming M, Chu Y, Moran B, Belytschko T, Lu Y, Gu L. Enriched element-free Galerkin methods for crack tip fields. Int J Numer Methods Eng 1997;40:1483-504.

[20] Rabczuk T, Bordas S, Zi G. On three-dimensional modelling of crack growth using partition of unity methods. Comput Struct 2010;88:1391-411.

[21] Goodman RE, Taylor RL, Brekke TL. A model for the mechanics of jointed rock. J Soil Mech Found Div 1968.

[22] Segura J, Carol I, Coupled HM. analysis using zero-thickness interface elements with double nodes. Part I: Theoretical model. Int J Numer Anal Methods Geomech 2008;32:2083-101. 
[23] Segura J, Carol I, Coupled HM. analysis using zero-thickness interface elements with double nodes-Part II: Verification and application. Int J Numer Anal Methods Geomech 2008;32:2103-23.

[24] Nick H, Matthäi S. Comparison of three FE-FV numerical schemes for singleand two-phase flow simulation of fractured porous media. Transport Porous Media 2011;90:421-44.

[25] Schlangen E, Garboczi E. Fracture simulations of concrete using lattice models: computational aspects. Eng Fract Mech 1997;57:319-32.

[26] Grassl P. A lattice approach to model flow in cracked concrete. Cement Concrete Compos 2009;31:454-60.

[27] Réthoré J, Borst R, Abellan MA. A two-scale approach for fluid flow in fractured porous media. Int J Numer Methods Eng 2007;71:780-800.

[28] Réthoré J, de Borst R, Abellan MA. A two-scale model for fluid flow in an unsaturated porous medium with cohesive cracks. Comput Mech 2008;42:227-38.

[29] Watanabe N, Wang W, Taron J, Görke U, Kolditz O. Lower-dimensional interface elements with local enrichment: application to coupled hydromechanical problems in discretely fractured porous media. Int J Numer Methods Eng 2012.

[30] Lamb AR, Gorman GJ, Elsworth D. A fracture mapping and extended finite element scheme for coupled deformation and fluid flow in fractured porous media. Int J Numer Anal Methods Geomech 2013;37:2916-63.

[31] Huang H, Long TA, Wan J, Brown WP. On the use of enriched finite element method to model subsurface features in porous media flow problems. Comput Geosci 2011;15:721-36.

[32] Bordas S, Rabczuk T, Zi G. Three-dimensional crack initiation, propagation, branching and junction in non-linear materials by an extended meshfree method without asymptotic enrichment. Eng Fract Mech 2008:75:943-60.

[33] Mohammadi S. Extended finite element method for fracture analysis of structures. Blackwell Publishing; 2008.

[34] Biot MA. General theory of three-dimensional consolidation. J Appl Phys 1941;12:155-64.
[35] Vardoulakis I, Stavropoulou M, Papanastasiou P. Hydro-mechanical aspects of the sand production problem. Transport Porous Media 1996;22:225-44.

[36] Barth TJ, Sethian JA. Numerical schemes for the Hamilton-Jacobi and level set equations on triangulated domains. J Comput Phys 1998;145:1-40.

[37] Stolarska M, Chopp D, Moës N, Belytschko T. Modelling crack growth by level sets in the extended finite element method. Int J Numer Methods Eng 2001;51:943-60.

[38] Gravouil A, Pierres E, Baietto M-C. Stabilized global-local X-FEM for 3D nonplanar frictional crack using relevant meshes. Int J Numer Methods Eng 2011;88:1449-75.

[39] Duflot M. A study of the representation of cracks with level sets. Int J Numer Methods Eng 2007;70:1261-302.

[40] Barsoum RS. Triangular quarter-point elements as elastic and perfectly-plastic crack tip elements. Int J Numer Methods Eng 1977; 11:85-98.

[41] Daux C, Moes N, Dolbow J, Sukumar N, Belytschko T. Arbitrary branched and intersecting cracks with the extended finite element method. Int J Numer Method Eng 2000;48:1741-60.

[42] Menk A, Bordas SPA. Numerically determined enrichment functions for the extended finite element method and applications to bi-material anisotropic fracture and polycrystals. Int J Numer Methods Eng 2010;83:805-28.

[43] Menk A, Bordas S. Influence of the microstructure on the stress state of solder joints during thermal cycling. New York: Ieee; 2009.

[44] Natarajan S, Bordas SP, Mahapatra DR. On numerical integration of discontinuous approximations in partition of unity finite elements, In: IUTAM symposium on multi-functional material structures and systems. 2010:297-304.

[45] Bordas S, Nguyen PV, Dunant C, Guidoum A, Nguyen-Dang H. An extended finite element library. Int J Numer Methods Eng 2007;71:703-32.

[46] Verruijt A. Theory of consolidation. Computational geomechanics. Springer; 1995;8-34. 